Findings of the US Research Needs Workshop on the Topic of Fusion Power

W. R. Meier, A. R. Raffray, R. J. Kurtz, N. B. Morley, W. T. Reiersen, P. Sharpe, S. Willms

September 21, 2009

Fusion Engineering and Design 
This document was prepared as an account of work sponsored by an agency of the United States government. Neither the United States government nor Lawrence Livermore National Security, LLC, nor any of their employees makes any warranty, expressed or implied, or assumes any legal liability or responsibility for the accuracy, completeness, or usefulness of any information, apparatus, product, or process disclosed, or represents that its use would not infringe privately owned rights. Reference herein to any specific commercial product, process, or service by trade name, trademark, manufacturer, or otherwise does not necessarily constitute or imply its endorsement, recommendation, or favoring by the United States government or Lawrence Livermore National Security, LLC. The views and opinions of authors expressed herein do not necessarily state or reflect those of the United States government or Lawrence Livermore National Security, LLC, and shall not be used for advertising or product endorsement purposes. 


\title{
Findings of the US Research Needs Workshop on the Topic of Fusion Power
}

\author{
W.R. Meier $^{1}$, A.R. Raffray ${ }^{2}$, R.J. Kurtz ${ }^{3}$, N.B. Morley ${ }^{4}$, \\ W.T. Reiersen ${ }^{5}$, P. Sharpe ${ }^{6}$, S. Willms ${ }^{7}$ \\ ${ }^{1}$ Lawrence Livermore National Laboratory, Livermore, CA USA \\ ${ }^{2}$ University of California, San Diego, San Diego, CA USA \\ ${ }^{3}$ Pacific Northwest National Laboratory, Richland, WA USA \\ ${ }^{4}$ University of California, Los Angles, LA, CA USA \\ ${ }^{5}$ Princeton Plasma Physics Laboratory, Princeton, NJ USA \\ ${ }^{6}$ Idaho National Laboratory, Idaho Fall, ID USA \\ ${ }^{7}$ Los Alamos National Laboratory, Los Alamos, NM USA
}

\begin{abstract}
The US Department of Energy, Office of Fusion Energy Sciences (OFES) conducted a Research Needs Workshop, referred to as ReNeW, in June 2009. The information developed at this workshop will help OFES develop a plan for US fusion research during the ITER era, roughly the next two decades. The workshop was organized in five Themes, one of which was Harnessing Fusion Power (or Fusion Power for short). The top level goal of the Fusion Power Theme was to identify the research needed to develop the knowledge to design and build, with high confidence, robust and reliable systems that can convert fusion products to useful forms of energy in a reactor environment, including a self-sufficient supply of tritium fuel. Each Theme was subsequently subdivided into Panels to address specific topics. The Fusion Power Panel topics were: fusion fuel cycle; power extraction; materials science; safety and environment; and reliability, availability, maintainability and inspectability (RAMI). Here we present the key findings of the Fusion Power Theme.
\end{abstract}

Key words: fusion power, fusion nuclear technology, materials, tritium, environment and safety

\section{Introduction}


In preparation for its strategic planning process, the US Department of Energy, Office of Fusion Energy Sciences (OFES) commissioned a Research Needs Workshop ( $\mathrm{ReNeW}$ ) focused on magnetic fusion energy (MFE) [1]. The objective of this activity was to provide OFES with the building blocks to develop an R\&D plan for fusion over the next $20+$ years. Essentially, the question posed was, "What R\&D, in addition to ITER, is require to prepare for a demonstration power plant?"

The workshop was organized in five Themes: Burning Plasma in ITER, HighPerformance Steady State, Plasma Material Interface, Fusion Power, and Magnetic Configuration. The focus of the Fusion Power Theme was the research needed to develop the knowledge to design and build, with high confidence, robust and reliable systems that can convert fusion products to useful forms of energy in a reactor environment, including a self-sufficient supply of tritium fuel.

\section{Fusion Power Panels}

Each Theme was subdivided into Panels to address specific topics. As illustrated in Fig. 1, Fusion Power Theme was organized into five Panels (as suggested in Ref. 2): Fusion Fuel Cycle, Power Extraction, Materials Science, Safety and Environment, and RAMI. The basic issues and top level R\&D needs for each Panel is summarized here.

Fusion Fuel Cycle: Fusion power plants, including DEMO, will burn deuterium and tritium (DT), producing 3.5 MeV alpha particles and $14 \mathrm{MeV}$ neutrons. Deuterium occurs naturally in vast quantities, but tritium is radioactive with a short half life and is not naturally available in quantities needed to fuel fusion plants. Fortunately, tritium can be produced by capturing fusion neutrons in lithium. Consequently, a lithiumcontaining tritium breeding blanket will be placed outside the thin wall that surrounds 
the plasma. We must determine how to manage the flow of tritium throughout the entire plant, including plasma fueling, breeding, recovery and containment.

Power Extraction: About $20 \%$ of the fusion power is absorbed as surface heat by components exposed to the plasma (first wall and divertors), while $\sim 80 \%$ is deposited volumetrically by neutrons in the breeding blanket. These components must reliably operate at a high enough temperature (e.g., > 600C) to efficiently produce electricity or hydrogen. We must develop the fusion chamber components that can achieve this capability.

Materials Science: The high-energy neutrons can displace atoms and produce helium and hydrogen by transmutations in the materials, degrading their properties and performance. Materials used in the plasma chamber structures, tritium breeding blankets, plasma diagnostics, and heating systems will suffer damage. We must understand the basic materials science phenomena of radiation damage and qualify components for use in fusion power plants.

Safety and Environment: Fusion energy has the potential to be environmentally attractive. Through careful choice of materials, the induced radioactivity lifetimes can be very short compared to those from fission reactors. All fusion materials should be recyclable to minimize the radwaste burden for future generations. Because of these and other inherent safety features, studies of possible fusion power plants indicate they will be safe and should have no need for a public evacuation plan. We must convincingly demonstrate the safety and environmental potential of fusion.

Reliability, Availability, Maintainability, and Inspectability (RAMI): DEMO must demonstrate a high enough availability for power producers to build a commercial fusion plant. Power producers cannot expect an ultimate fusion power plant availability of $80 \%$ (or more) if DEMO cannot demonstrate a $50 \%$ or higher availability. Achieving this DEMO availability goal will require reliability in component design, design 
integration for RAMI, high maintainability, and systems to monitor and inspect components. We must develop and qualify methods and capabilities needed to achieve RAMI objectives.

\section{Research Thrusts}

Base on work leading up to the workshop (including community workshops that focused on individual Themes) and presentations and discussions at the June workshop, the fusion community developed 18 research thrusts to encompass the research needs identified by the five Themes and their topical Panels. These are reported in detail in Ref [2]. While the research needs for the Fusion Power theme are address in a variety of Thrusts, there were three primary thrusts that evolved from the Fusion Power Panels. They were numbered Thrusts 13-15 in and are briefly described here.

\subsection{Establish the Science and Technology for Fusion Power Extraction and Tritium Sustainability (Thrust-13)}

Harnessing fusion power for useful purposes will require significant advances in the understanding of fusion fuel cycle and power extraction. ITER and later DEMO (an energy demonstration reactor) will be significant extensions beyond present experience, requiring the successful operation of systems that currently have never been fabricated, demonstrated, or tested in a relevant in-service environment. The primary focus of this Thrust is to address this situation by developing the science base and technological readiness for safe and reliable: 1) power handling and extraction, 2) operation of the fusion fuel cycle, and 3) tritium breeding and extraction.

As a practical energy source, a fusion power plant must create the tritium fuel it uses (by capturing fusion neutrons in the element lithium) and operate at high temperature so that the fusion energy can be converted efficiently to electrical power or 
other end uses. This Thrust aims to develop the scientific foundations of practical, safe and reliable processes and components that 1) harvest the heat produced by fusion, 2) create and extract the tritium from lithium, and 3) manage (radioactive) tritium that circulates in the plant. A continuous effort of experimental research and predictive model development, focused on the phenomena and interactions occurring in fusion nuclear components, is essential, both to prepare for next steps in burning plasma physics research as well as to accelerate progress toward practical fusion energy as the ultimate goal.

Key Issues:

- How can fusion power be extracted from the complex structures surrounding the burning plasma? Can these structures be engineered to operate reliably in this extreme environment?

- How do we contain and efficiently process the tritium fuel in a practical system? Can this unprecedented amount of mobile tritium be accounted for accurately?

- How should lithium-bearing materials be integrated into power extraction components to generate tritium fuel to replace that burned in the plasma? Can simultaneous power extraction and fuel sustainability be achieved?

\section{Proposed Actions:}

- Perform fundamental research to establish the scientific parameters necessary to address the issues. An example activity is the exploration of tritium chemistry, heat transfer, and magnetic field interactions in lithium-bearing liquid metal coolants.

- Perform multiple-effect studies to understand the combined impact of the operating conditions and component complexity typical of a fusion environment. An example activity is utilizing the ITE R burning plasma as a test environment to perform 
tritium breeding and power extraction experiments with relevant materials, instrumentation, component designs, and operating temperatures with necessary ancillary systems.

- Perform integrated experiments to characterize the complete effect of fusion conditions and facility performance. An example would be construction and operation of a Fusion Nuclear Science Facility (FNSF) to perform testing that resolves the remaining gaps stemming from the effects of significant surface heat flux and neutron irradiation over a long period of time in concert with all other fusion environmental conditions.

- Develop the accompanying theory and predictive models necessary to understand and apply the experimental results, and collect reliability, tritium accountability and safety data at all stages.

The flows of power and tritium associated with this Thrust are shown on Figure 2.

\subsection{Develop the Material Science and Technology needed to Harness Fusion}

\section{Power (Thrust-14)}

For fusion to find its way into the energy marketplace it must compete economically with other energy options, and it must be developed as a safe and environmentally acceptable energy source, particularly from the viewpoint of radioactivity. Achieving acceptable performance for a fusion power system in the areas of economics, safety and environmental acceptability is critically dependent on performance, reliability and lifetime of the first-wall, blanket and divertor systems, which are the primary heat recovery, plasma purification, and tritium breeding systems. Design and performance of these key components are in turn critically dependent on the properties and characteristics of the structural materials. Since these are primary 
fusion power feasibility issues and since resources are limited, the main focus of recent fusion materials research has been on structural materials. Based on safety, waste disposal and performance considerations, the selection of structural materials for blanket and divertor applications is largely limited to reduced activation ferritic/martensitic (RAF/M) steels, dispersion strengthened ferritic alloys, tungsten alloys and silicon carbide composites. However, it is fully recognized that a host of other materials, such as plasma facing, breeding, shielding, insulating, superconducting and diagnostic materials, must also be successfully developed for fusion to be a technologically viable power source. A comprehensive research program must address the specific materials science issues associated with each of these materials systems. This Thrust provides the principal source of knowledge on fundamental materials behavior and radiation effects to support development of radiation-resistant materials needed several of the other Thrusts described in the workshop report.

Fusion materials and structures must function for a long time in a uniquely hostile environment that includes combinations of high temperatures, reactive chemicals, high stresses, and intense damaging radiation. Ultimately, we need to establish the feasibility of designing, constructing and operating a fusion power plant with materials and components that meet demanding objectives for safety, performance and minimal environment impact.

Key Issues:

- What thermal, mechanical, and electrical properties are needed to meet fusion objectives?

- How does radiation damage affect the properties of materials? 
- How do synergistic effects involving radiation damage, high temperatures, high stresses and corrosion phenomena affect the feasibility of operating a fusion power plant?

\section{Proposed Actions:}

- Improve the performance of existing and near-term materials, while also developing the next generation of high-performance materials with revolutionary properties.

- Understand the relationship between material strength, ductility and resistance to cracking. Design materials with exceptional stability, high-temperature strength and radiation damage tolerance.

- Understand how interactions with the plasma affect materials selection and design. Establish the scientific basis to control the corrosion of materials exposed to aggressive environments.

- Develop the technologies for large-scale fabrication and joining.

- Determine the underlying scientific principles to guide discovery of revolutionary high-performance materials while minimizing radioactive waste and maximizing recycling.

- Develop and experimentally validate predictive models describing the behavior and lifetimes of materials in the fusion environment.

- Establish a fusion-relevant neutron source to enable accelerated evaluations of the effects of radiation-induced damage to materials.

- Implement an integrated design and testing approach for developing materials, components, and structures for fusion power plants.

- Use a combination of existing and new nonnuclear and nuclear test facilities to validate predictive models and determine the performance limits of materials, components and structures. 
The summary, the purpose of Thrust 14 is to provide the fundamental materials science and technology necessary to enable design, construction and operation of a fusion power plant. In this Thrust the basic materials property information and models of materials behavior in the harsh fusion environment will be developed. This information provides the foundation for selecting, developing and qualifying materials to meet design requirements. Research and development of plasma facing and blanket components will carry out in progressively more integrated tests that use the materials and models developed in this Thrust. This is a highly iterative process since evaluation of materials performance from integrated tests will be used to refine materials selection and processing pathways, which in turn determines the available design window.

\subsection{Create Integrated Designs and Models for Attractive Fusion Power Systems}

\section{(Thrust 15)}

A broad-based effort is needed to understand, model and evaluate the various components, subsystems and processes that must be integrated to create an acceptable fusion power plant (including closing the tritium fuel cycle and efficiently harnessing the fusion power). This will provide understanding of very complex interrelated behavior of systems that is otherwise difficult to anticipate, and could dramatically reduce the cost and risk of future $R \& D$. Thrust 13 and 14 research focuses on the fusion fuel cycle, power extraction and materials, and modeling tools that will be developed and used to investigate specific aspects supporting this R\&D. Thrust 15 focuses on integration; it includes conducting integrated advanced design studies focused primarily on DEMO, but also on nearer term fusion nuclear facilities, and integrating simulation models. The integrated models will simulate increasingly complex, coupled aspects of 
the fusion plant with a focus on the plasma chamber, breeding blanket, fuel cycle and power extraction.

Capturing the energy released by a burning plasma and converting it to a useful power flow in a safe, reliable, and sustainable manner requires the successful integration of many systems and physical processes. This Thrust includes two primary aspects: 1) conducting advanced design studies; and 2) developing integrated predictive models. Detailed advanced design studies bring to light key design trade-offs and constraints within an integrated system for DEMO. For example, maximizing power cycle thermal efficiency requires operation at high temperatures, with upper limits set by coolant and structure compatibility, thermal stress, material behavior and plasma thermal loading considerations. Identifying serious limiting factors and design issues can guide research toward high-leverage and high-payoff issues while minimizing program risk. Such detailed advanced design studies also allow the assessment of integrated safety, environmental and RAMI (reliability, availability, maintainability and inspectability) issues for fusion. Advanced design activities are also essential in evaluating alternatives for a Fusion Nuclear Science Facility (FNS F), which is an important and necessary step on the path to fusion energy. Integrated models are used to help reveal important science and technology interrelationships and interpret the results of fusion experiments and component tests, thereby reducing the risks and costs in the development of fusion nuclear systems. They will also support the advanced design studies for future fusion facilities.

Key Issues:

- What advanced design studies are needed to identify system integration issues; optimize facility configuration; extend the operating parameter space for future fusion facilities that meet availability, maintainability, safety and environmental 
goals on the path to fusion power; and guide the R\&D on high-leverage, highpayoff issues?

- How can separate physics, nuclear, and engineering models be most effectively coupled to address highly integrated fusion system behavior?

\section{Proposed Actions:}

- Determine and improve essential aspects of fusion energy through advanced design and integration activities for DEMO, including:

- Optimizing the integrated configuration and maintenance approach to minimize risk and achieve the availability, maintainability, safety and environmental requirements for DEMO to be attractive.

- Determining the scientific basis for sustainable fusion power and identifying research efforts to close the knowledge gap to DEMO.

- Evaluate, through advanced design and system studies, alternative configurations and designs for FNS F and the extent to which they address the research requirements.

- Develop predictive modeling capability for nuclear components and associated systems that are science-based, well-coupled, and validated by experiments and data collection.

- Extend models to cover synergistic physical phenomena for prediction and interpretation of integrated tests (e.g., ITE R Test Blanket Module - TBM) and for optimization of systems.

- Develop methodologies to integrate with plasma models to jointly supply key first wall and divertor temperature and stress levels, electromagnetic responses, surface erosion, etc. 


\section{Summary}

The US fusion program has recently completed an evaluation of research needed to prepare for DEMO, including the entire range of activities from plasma physics to fusion power systems. The research in support of fusion nuclear science and technology was primarily covered in the Harnessing Fusion Power Theme of the workshop and was combined into three broad research Thrusts: one dealing with the fusion power extraction and fuel cycle; one focused on material science and technology, and the third encompassing integrated design studies and integrated modeling. Overall, 18 research Thrusts were proposed and these will form the building block from which OFES will construct its strategic R\&D plan for the next 20-30 years.

\section{Acknowledgements}

The authors wish to acknowledge the hard work and contributions of all the members of the five Panels that comprised the Fusion Power Theme. Portions of this work performed under the auspices of the U.S. Department of Energy by Lawrence Livermore National Laboratory under Contract DE-AC52-07NA27344.

\section{References}

[1] "Research Needs for Magnetic Fusion Energy Sciences- Report of the Research needs Workshop (ReNeW), June 8-12, 2009” (Richard Hazeltine, Chair), DOE/OFES report (August, 2009).

[2] M. Greenwald et al., "Priorities, Gaps and Opportunities: Towards A Long-Range Strategic Plan For Magnetic Fusion Energy.” http://www.ofes.fusion.doe.gov/FESACPlanningReport.pdf 
\# 06-022

\section{Corresponding Author:}

Wayne Meier

LLNL

L-637

P.O. Box 808

Livermore, CA 94583 USA

Email: meier5@LLNL.gov

Phone: 1-925-422-8536

Fax: 1-925-424-6401 
\# 06-022

\section{Figure captions}

Figure 1. The Harnessing Fusion Power Theme was organized into five topics (Panels): Fusion Fuel Cycle, Power Extraction, Materials Science, Safety and Environment, and RAMI.

Figure 2. Schematic of power and tritium flow covered in Thrust 13. 


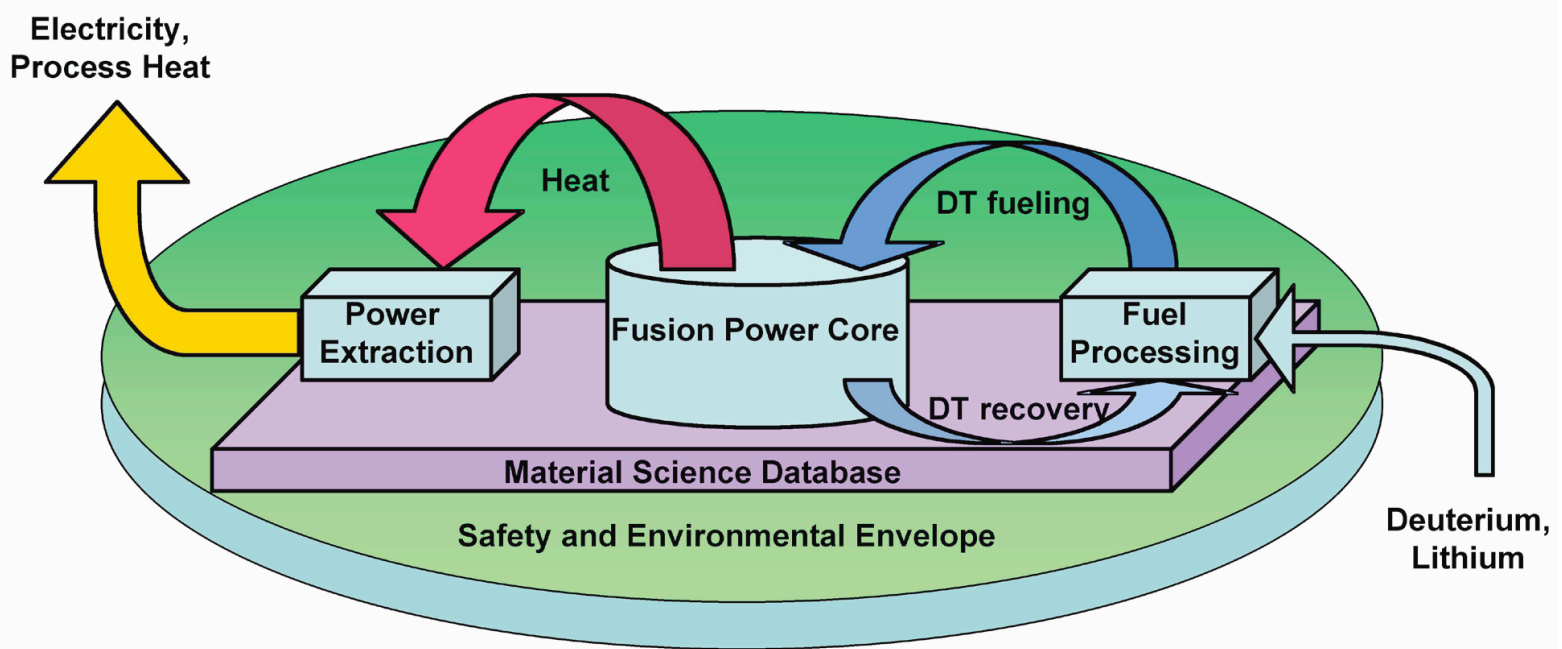

Reliability, Availability, Maintainability, Inspectability (RAMI) Foundation

Figure 1. The Harnessing Fusion Power Theme was organized into five topics (Panels): Fusion Fuel Cycle, Power Extraction, Materials Science, Safety and Environment, and RAMI. 


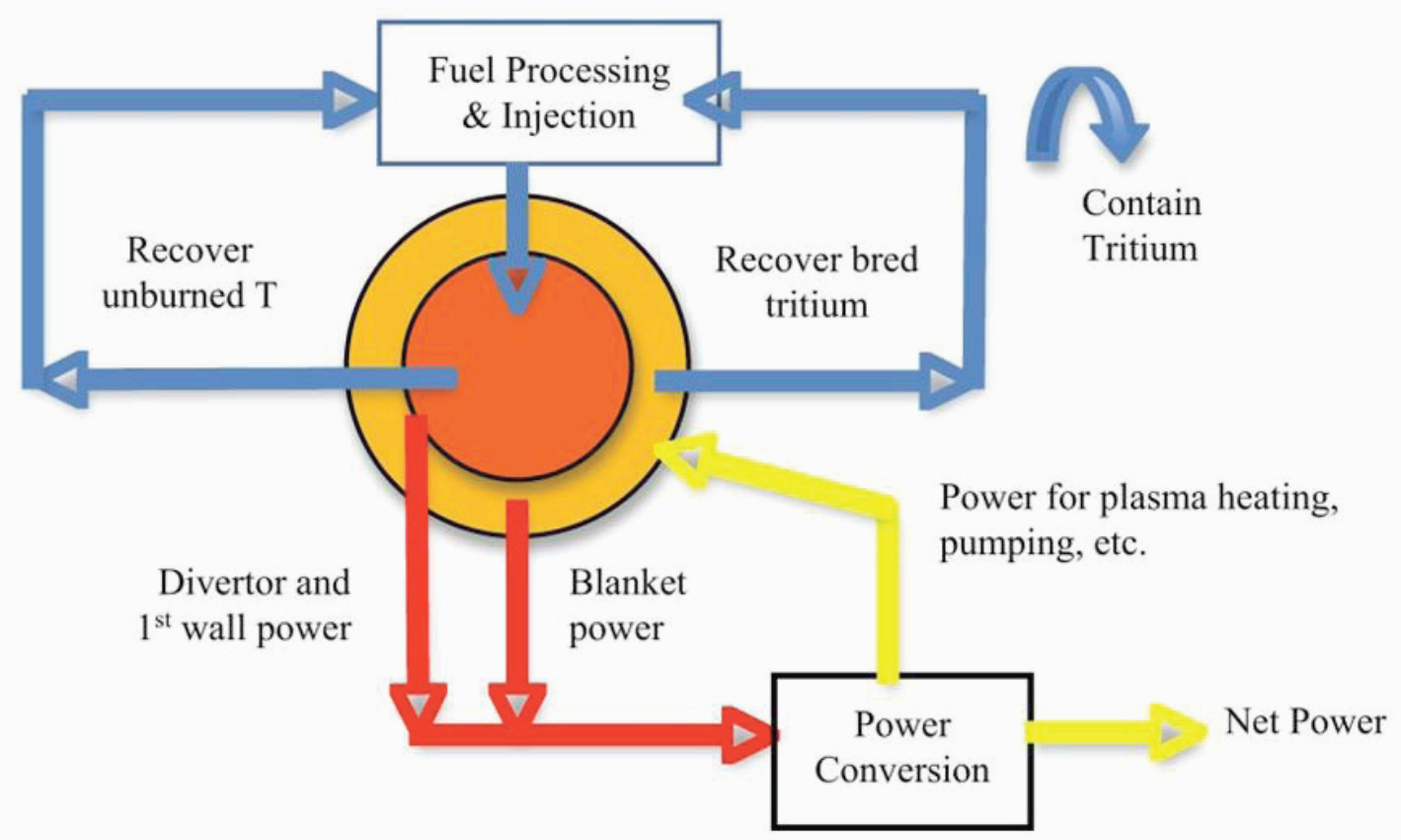

Figure 2. Schematic of power and tritium flow covered in Thrust 13. 